\title{
The care pathway for hip fracture from acute phase to rehabilitation
}

\author{
Paolo Sciattella, Valeria Belleudi, Paola Colais, Mirko Di Martino, Luigi Pinnarelli, Francesca Mataloni*, Marina Davoli, \\ Danilo Fusco
}

From Health Services Research: Evidence-based practice

London, UK. 1-3 July 2014

\section{Background}

Hip fractures are the leading cause of hospitalization for injuries among the elderly population and have a substantial impact on both the patient and the healthcare system. The care pathway for a person with a disability goes through a set of integrated and multidisciplinary activities and care interventions. Starting with timely surgery in the acute phase, followed by adequate rehabilitation assistance and ending with territorial assistance.

\section{Aims}

The aim of the study is to evaluate the association between early surgery and access to rehabilitation of elderly patients with hip fracture.

\section{Materials and methods}

We identified elderly patients hospitalised for hip fracture, between 1 January 2012 and 31 October 2012 using Hospital Information System (HIS) of the Lazio region. The outcome considered was the access to rehabilitation within 60 days from the date of hospital discharge. Rehabilitation access was derived from Admission/Discharge Rehabilitation Report (ADRR), for hospital rehabilitation, and from Residential Rehabilitation Information System (RRIS).

We considered clinical variables, residence and level of education as potential risk factors of the outcome. The factors significantly associated with outcome (gender, age, comorbidities, residence, level of education, hospital stay) were selected by a bootstrap stepwise procedure and their Risks Ratio (RR) were estimated through a multivariate regression model.

Department of Epidemiology, Lazio Region Health Service, Rome, Italy

\section{Results}

We selected 5,030 patients aged 65+ hospitalised for hip fracture in Lazio region, 59\% with an access to rehabilitative care within 60 days from discharge. The access to rehabilitation was less likely for older patients (adjusted $\mathrm{RR}=0.74, \mathrm{p}<0.001$ ), for patients with a longer acute event hospital stay (adjusted $R R=0.74, p<0.001$ ) and for patients with senile dementia (adjusted $R R=0.66, p<0.001$ ). By contrast, the probability was higher for patient who had surgery within $48 \mathrm{~h}$ (adjusted $\mathrm{RR}=2.60, \mathrm{p}<0.001$ ) and for residents in Rome (adjusted $\mathrm{RR}=1.24 \mathrm{p}<0.001$ ).

The level of education seems to be negatively associated with the outcome (adjusted $R R=0.79 p<0.05$ for patients with high level), probably because patients with a higher level of education could more easily obtain access to private services.

\section{Conclusions}

Despite the fact that following hip fracture rehabilitation should involve all patients, in the Lazio region the percentage of access to rehabilitation within 60 days from hospital discharge is $59 \%$. Access to rehabilitation is strongly dependent on age, clinical characteristics and acute hospital care; in particular, waiting time for surgical treatment is strongly correlated with access to rehabilitation. This could suggest that the care pathway evaluation should take into account the association between the acute and rehabilitation phase.

Published: 7 July 2014

doi:10.1186/1472-6963-14-S2-P74

Cite this article as: Sciattella et al.: The care pathway for hip fracture

from acute phase to rehabilitation. BMC Health Services Research 2014 14(Suppl 2):P74. 